\title{
Experimental study of impact on SMC composites used in the automotive industry
}

\author{
Gabriel Centeno, Francisco G. Benítez and \\ Johan P. Wideberg*
}

Transportation Engineering, Escuela Superior de Ingenieros, Universidad de Sevilla, Av. Camino de los Descubrimientos s/n, Sevilla 41092, España

E-mail: gabrielcenteno@esi.us.es

E-mail: benitez@us.es

E-mail: wideberg@esi.us.es

${ }^{\star}$ Corresponding author

\begin{abstract}
The signal from a piezoelectric sensor received by a data acquisition system was used to record the temporal evolution of the force transmitted by an impactor on specimens of an SMC composite. A high-speed motion analyser recorded the sequence of images of the impact and fracture of the material. The subsequent processing of this sequence using an image analysis programme provided data for the calculation of various energy magnitudes. The behaviour under impact of this material was then characterised on the basis of these energy magnitudes and the temporal evolution of the force.
\end{abstract}

Keywords: SMC; impact of SMC; experimental testing.

Reference to this paper should be made as follows: Centeno, G., Benítez, F.G. and Wideberg, J.P. (2007) 'Experimental study of impact on SMC composites used in the automotive industry', Int. J. Vehicle Design, Vol. 44, Nos. 3/4, pp.282-292.

Biographical notes: Gabriel Centeno is a $\mathrm{PhD}$ candidate at the Engineering Institute of the University of Seville. His areas of interest include impact of composite materials.

Francisco G. Benítez is a Professor at the University of Seville Transportation Engineering group. He first joined the University in 1985. He finished his $\mathrm{PhD}$ at the Politechnical University in Madrid (Spain) in 1981. He was appointed Research Fellow at the Engineering Science Department of Oxford University from 1981 to 1983 and hold a Fulbright Scholarship at the California Institute of Technology from 1984 to 1985 . He teaches courses in transportation, vehicles and railways engineering. His research interests are inscribed in the area of transport modelling, transmissions and impact experimental analysis.

Johan P. Wideberg is a Professor at the University of Seville Transportation Engineering group. He first joined the University in 1992 after working at ABB's research laboratory in Switzerland. After finishing his PhD in 1995 he joined the United Technologies Research Centre where he worked in the structural integrity group. Since 1999 he teaches courses in the subject areas of vehicle technology and transportation engineering. His research relates to 
vehicle dynamics, intelligent transportation systems and impact simulation of composite materials. He is also the founder and Editor-in-chief of the International Journal of Vehicle System Modelling and Testing.

\section{Introduction}

Composite materials are today widely used in industry in general and SMC materials in particular are massively used in the automotive industry, especially in the manufacture of industrial vehicle bodies such as truck cabins. See for instance Marsh (2003) for a general introduction and history.

The trend is for composite materials to more and more substitute metals in certain industrial applications. This is because, while their resistance is lower, their resistance/weight ratios are very high, making them attractive alternatives to metal. This and their relatively low cost and ease of manufacture and transformation, have led to them attaining very high levels of productivity.

While metal (e.g. steel) components absorb the energy of an impact by plastic deformation, composite materials do so by fracture of the fibres and rupture of the matrix. The principal cause of structural failure of SMC materials in automotive applications is due to the low-energy impacts generated by small collisions. This is the reason for the interest in characterising this type of material's response to these dynamic demands.

The objective of this paper is to study the behaviour of an SMC composite subjected (in this case automotive panels) to low-energy impact loads and the subsequent discussion of the failure mechanisms.

\section{State of the art}

Experimental studies of SMC composites are being carried out in numerous public, corporate and university research centres throughout the world. In the EU in particular, the importance of these studies is reflected in the creation of business alliances such as the 'European Alliance for SMC', formed by companies from Germany, UK, France, Spain and Italy in order to coordinate and accelerate the development of improved materials and new products.

One of the techniques used in these trials is impact by fall under gravity onto specimens of an SMC composite. An outstanding work in this area is that of Lee et al. (1999). In their impact trials, they studied the influence of the mass and shape of the impactor, the speed of impact and the thickness of the material. They contrasted the experimental results with a model constructed using the LS-DYNA 3D software package. Other SMC studies are that of Morozov et al. (2003) and Oldenbo et al. (2003), although their trials were static. They contrasted the results of three-point bending of the material with a numerical model constructed using FEA tools.

There has been considerable research on impact behaviour in composite materials such as SMCs as well as other types of composites. Comprehensive reviews can be found in this papers by Abrate (1994), Corbett et al. (1996) and Richardson and Wisheart (1996). 
With respect to studies of impacts on composite materials in general, Aslan et al. (2003) used a custom-designed device to carry out low-speed (constant $3 \mathrm{~m} / \mathrm{s}$ ) impacts. They studied the effect of varying the mass of the impactor and used finite element modelling (with the 3D IMPACT software package) to contrast the experimental results. Also in this line, Jiang and Shu (2004) performed transverse impacts which they analysed using 3D finite element modelling to determine the distribution of stresses. Dear and Brown (2003) used a servo-hydraulic device to study different degrees of deformation and damage by penetration under impact. Finally, Abrate (2001) presented a classification and theoretical discussion of the types of model that can be used to analyse impact dynamics.

\section{Experimental set-up}

Low-energy impact trials were conducted using a set-up consisting of various elements, the most important of which was a tower for the fall under gravity. The other devices included a data acquisition system and a high-speed motion analyser.

The different devices were appropriately interconnected to obtain the data from each trial. The information from the different measurement and data capture devices was fed to a personal computer, on which the results of each trial were processed.

\subsection{Drop tower}

The drop tower was of our own construction. Two columns guide the fall of a head beneath which is the impactor body. A piezoelectric sensor, housed inside the impactor body, is responsible for providing the data on the temporal evolution of the impact force. The hoisting system is a hand-operated crane which raises the head to the desired height of the trial. The head is released by cutting off the current to an electromagnet at the end of the hoisting cable and attached magnetically to a metal plate on the top of the head. The impactor strikes the specimen which is placed between supports on a coordinate grid table. An anti-rebound system avoids the possibility of the head impacting on the specimen a second time.

The tower permits the head to be raised to different heights, which therefore correspond to different initial kinetic energies at impact. The principal characteristics of the tower are: mass of the combined head-impactor $9.7 \mathrm{~kg}$; maximum height of fall $2.23 \mathrm{~m}$; maximum speed at impact $6.6 \mathrm{~m} / \mathrm{s}$; maximum energy at impact $212 \mathrm{~J}$ and a frontal spatial window of the trials of $400 \mathrm{~mm}$.

\subsection{Data acquisition system}

The data acquisition system is responsible for recording the temporal evolution of the force of impact. To this end, it includes a piezoelectric sensor, a load attenuator, a single-channel signal amplifier, a connector box and a data acquisition card.

The data acquisition is controlled using the LABVIEW program from a personal computer equipped with the appropriate data acquisition card. Data from the equipment is recorded at a rate of $20 \mathrm{kHz}$ and is stored in files that are subsequently processed with the program MATLAB to give the graphs of the temporal evolution of the force in the impactor versus the time during the impact process. 


\subsection{Motion analyser}

A KODAK EKTAPRO HS 4540 high-speed motion analyser allows the capture of up to 16,000 images at a rate of 40,500 images per second, although, in the present trials, 1024 full-screen images were captured at a rate of 4500 images per second. The images are stored in the internal memory of the device itself.

The image capture process is started by a trigger signal from a position sensor (see Figure 1).

Figure 1 Schematic diagram of the impact trial apparatus

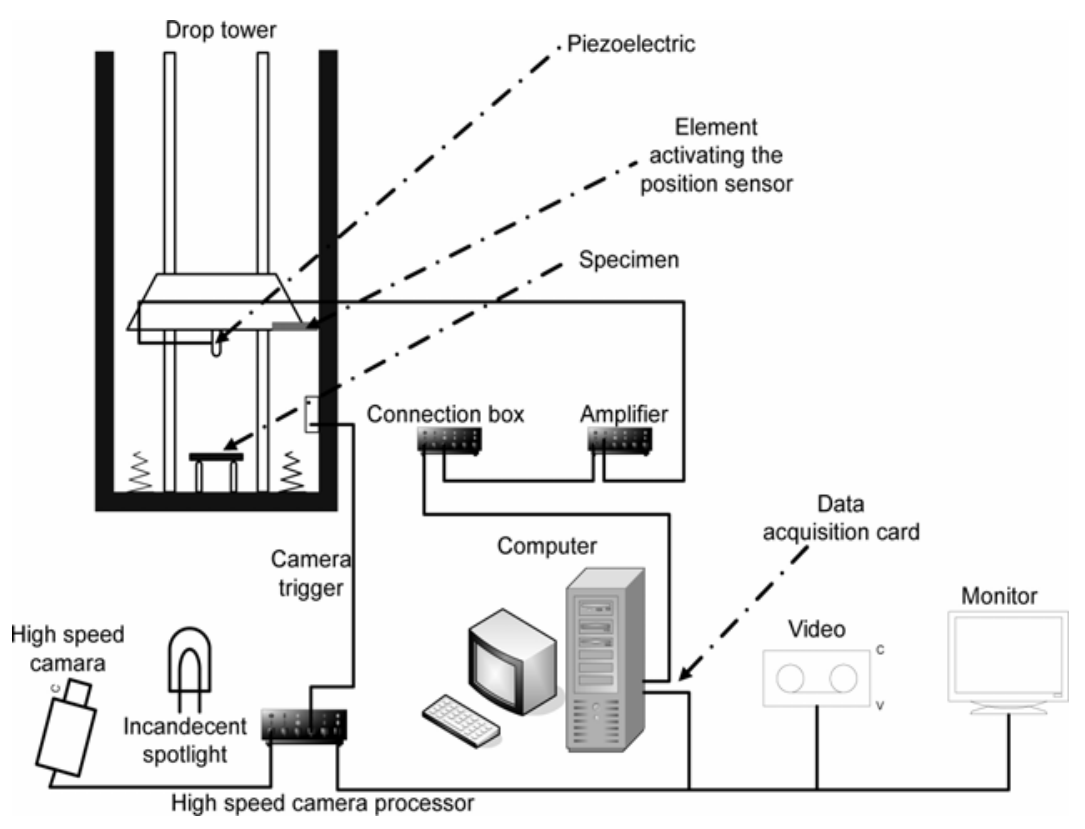

The process requires an intense, non-fluctuating light source, which is provided by a $1000 \mathrm{~W}$ incandescent spotlight. There are also close-up lenses and an objective lens for the camera.

The images are downloaded to the computer through an interface and subsequently processed by means of an image analyser that gives the speed and position at each instant of the sequence. From the evolution of the speed one can determine the variations in kinetic energy which, assuming zero friction of the falling head with the columns of the tower, correspond to the temporal evolution of the energy absorbed by the specimen. The evolution of the position of the head allows the indirect measurement of the deflection of the specimen. From these data, one obtains representations of the different energy magnitudes.

\section{Dynamic model}

In the following, we present the dynamic model of the experiment from the instant of impact. The free falling head, of mass $M$, is subject on impact to a time-dependent vertical force that represents the force transmitted to the specimen (Figure 2). 
Figure 2 Dynamic model and photograph of the head
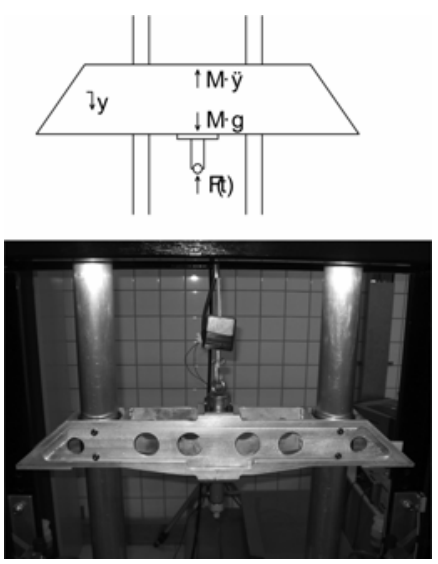

The problem's initial conditions are an initial velocity equal to the speed of impact $v=v_{0}$ for a reference time and position $t=0$ and $y=0$. From the balance of forces, one has:

$$
M g-F(t)-M \ddot{y}(t)=0
$$

Integrating twice, the position of the head with respect to time is:

$$
y(t)=y(0)+v_{0} t+\frac{1}{2} g t^{2}-\frac{1}{M} \int_{0}^{t} \int_{0}^{t} F(t) \mathrm{d} t^{2}
$$

The kinetic (3) and potential (4) energies of the head are:

$$
\begin{aligned}
& E_{c}(t)=\frac{1}{2} M(\dot{y}(t))^{2} \\
& E_{p}(t)=-M g y(t)
\end{aligned}
$$

The maximum energy of the head during impact is its initial kinetic energy calculated at $t=0, y=0$. The various energy magnitudes starting from that instant are calculated from the evolution of the kinetic energy after impact.

\section{Test material}

The test material was an SMC composite with the following properties:

Density

Young's modulus

Apparent elastic limit

Tensile resistance

Deformation at rupture

Proportion of glass fibre

$$
\begin{aligned}
& \rho=1950 \pm 30 \mathrm{~kg} / \mathrm{m}^{3} \\
& E=11156.11 \mathrm{MPa} \\
& \sigma_{E}=56.86 \mathrm{MPa} \\
& \sigma_{U}=77.79 \mathrm{MPa} \\
& 0.68 \%
\end{aligned}
$$

Minimum 20\%

The specimens were machined to $100 \times 40 \times 4.8 \mathrm{~mm}$. Figure 3 shows the state of these specimens after the trials. 
Figure 3 Specimens after the impact trials

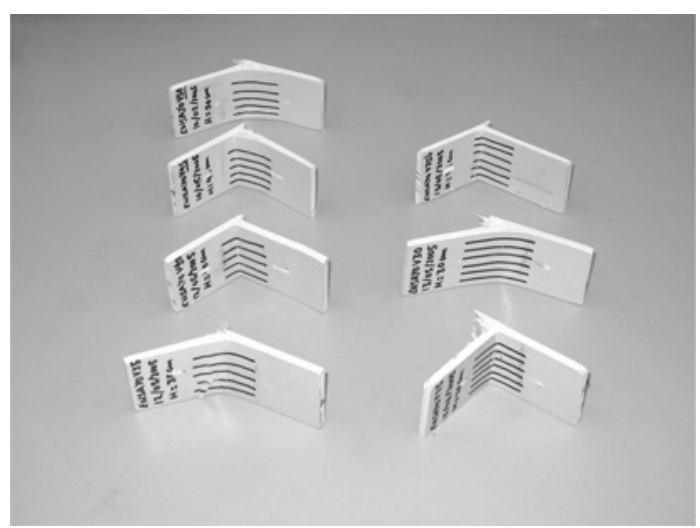

\section{Trials and results}

Trials were carried out at different heights of impact, from 50 to $25 \mathrm{~cm}$ at $5 \mathrm{~cm}$ intervals. The trials are denoted as V50, V45, V40, V35, V35b, V30 and V25, where the number represents the height of fall in $\mathrm{cm}$.

In each trial, the temporal evolution of the force in the impactor was recorded. Figure 4 shows this evolution for the trials at 50 and $25 \mathrm{~cm}$ height of fall.

Figure 4 The evolution of the force with respect to time in the trials V50 and V25
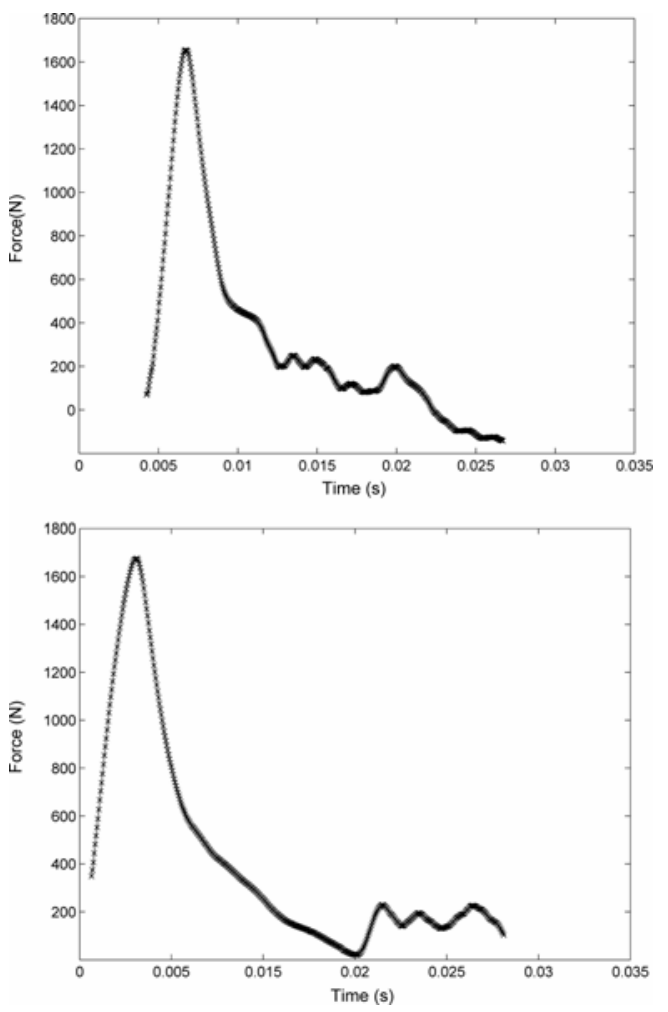
The most interesting parameter in the evolution of the force in the impactor is its maximum or peak value. The following table lists this value for each of trials.

\begin{tabular}{llllllll}
\hline Test & $V 50$ & $V 45$ & $V 40$ & $V 35$ & $V 35 b$ & $V 30$ & $V 25$ \\
\hline $\begin{array}{l}\text { Height of fall } \\
\begin{array}{l}\text { Maximum } \\
\text { force }\end{array}\end{array}$ & $50 \mathrm{~cm}$ & $45 \mathrm{~cm}$ & $40 \mathrm{~cm}$ & $35 \mathrm{~cm}$ & $35 \mathrm{~cm}$ & $30 \mathrm{~cm}$ & $25 \mathrm{~cm}$ \\
\hline
\end{tabular}

Figure 5 shows the sequence of images for one of the trials. The interval between frames is $0.0011 \mathrm{~s}$.

Figure 5 Sequence of images at $0.0011 \mathrm{~s}$ intervals of one of the trials

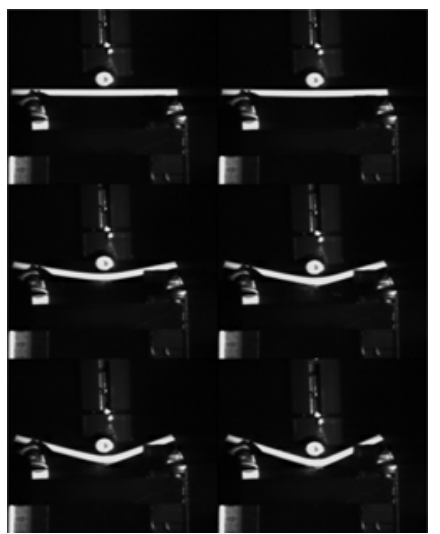

The evolution of the energy absorbed by the specimen was determined from the energy analysis performed in processing the image sequences. Figure 6 shows, for each trial, this evolution versus the time after impact and versus the deflection of the specimen.

One observes in the first of these plots that the energy absorbed by the specimens increases with respect to time, although the slope of the curve decreases. This is because, as the fibres fracture and the matrix of the specimen breaks, the latter is capable of absorbing less energy per differential time interval. One also observes that the slopes of the curves in the first instants after impact are steeper the greater the height of fall. This implies that also, just after impact, more energy is absorbed by the specimen, the greater the height of fall.

For the graph of the energy absorbed by the specimen versus deflection, however, there is much overlap of the curves for the different heights of impact. They all tend to a straight line behaviour, that is, to an approximately constant slope. One hence deduces that, for the range of heights used in the present study, the absorbed energy accumulated by the specimen during the process of impact depends linearly on the deflection of the specimen, regardless of the height of impact.

Figure 7 shows the result of the linear regression analysis of the absorbed energy versus deflection data for all the trials without regard for the height of impact. Also plotted are the $95 \%$ confidence limits on each side of the linear regression best fit. The expression for the linear regression, where the units of the deflection $f$ and the absorbed energy $E_{\text {abs }}$ are $m$ and $J$, respectively, is:

$$
E_{\mathrm{abs}}=1.4736+358.8658 f
$$


Figure 6 Evolution of the energy absorbed by the specimen versus time and versus the deflection of the specimen
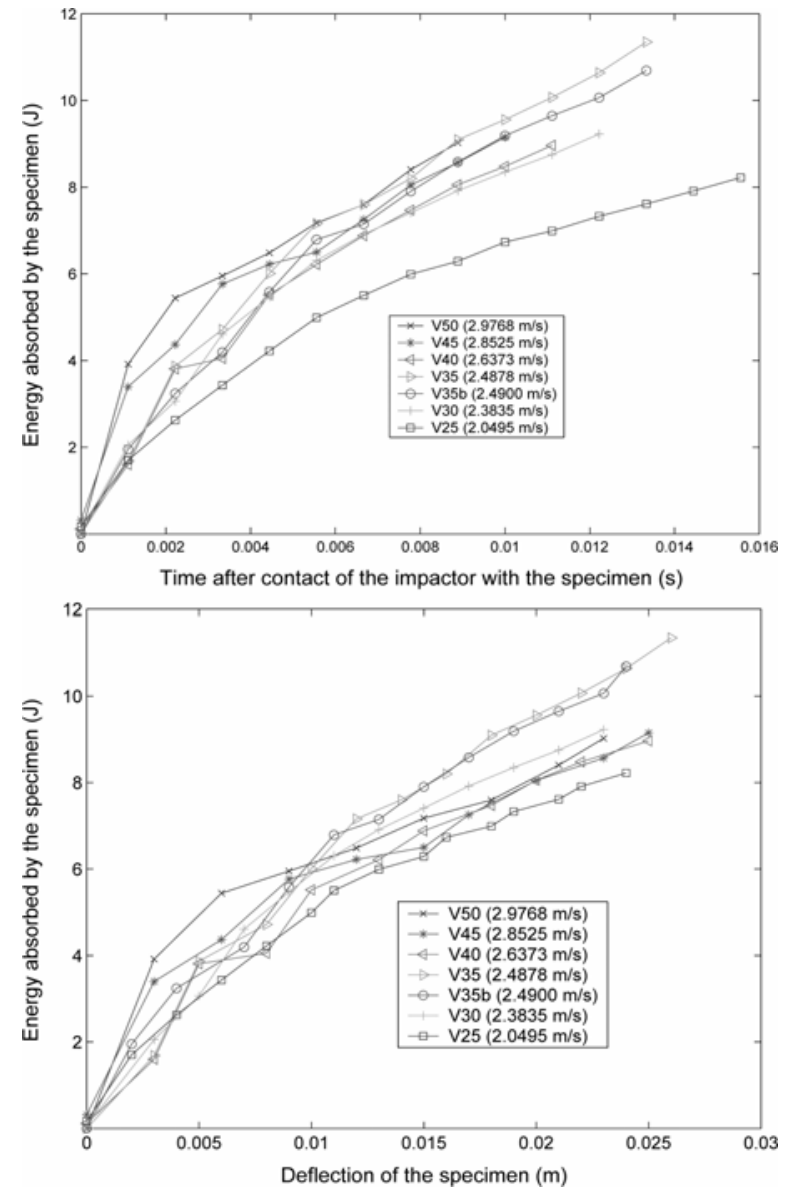

Figure 7 Linear regression analysis of all the trials

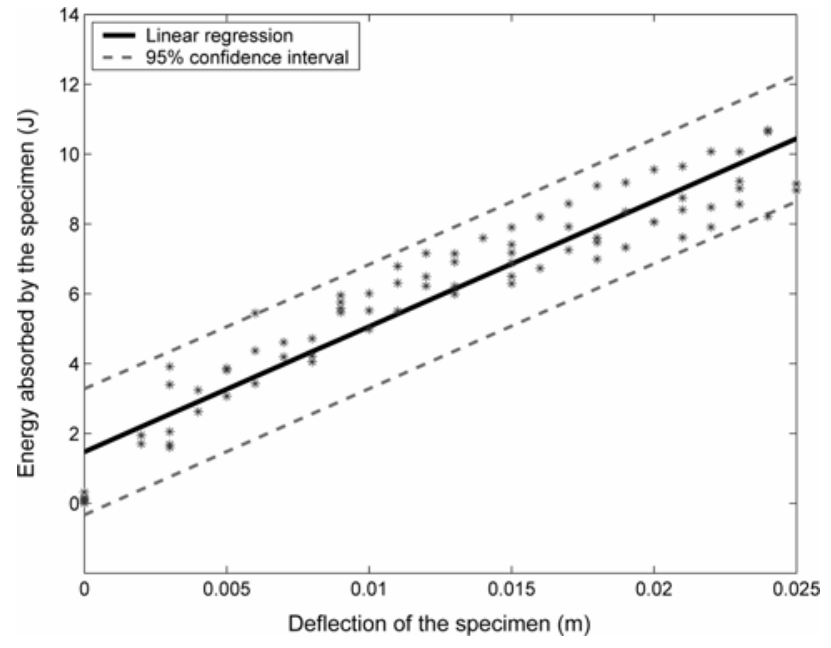


This Equation (5) represents the energy behaviour of the material as a function of the deflection, which, as seen above, is independent of the impact speed in the range of speeds of the present study.

In Figure 8, the first plot shows the energy absorbed in each interval of the sequence of images versus the deflection of the specimen. The second plot shows the quotient between the energy absorbed in each interval of the sequence and the incremental deflection of the specimen again versus the deflection of the specimen.

Figure 8 Energy absorbed by the specimen in each time interval of the image sequence and energy absorbed in each time interval divided by the increment in the deflection of the specimen, both plotted versus the deflection
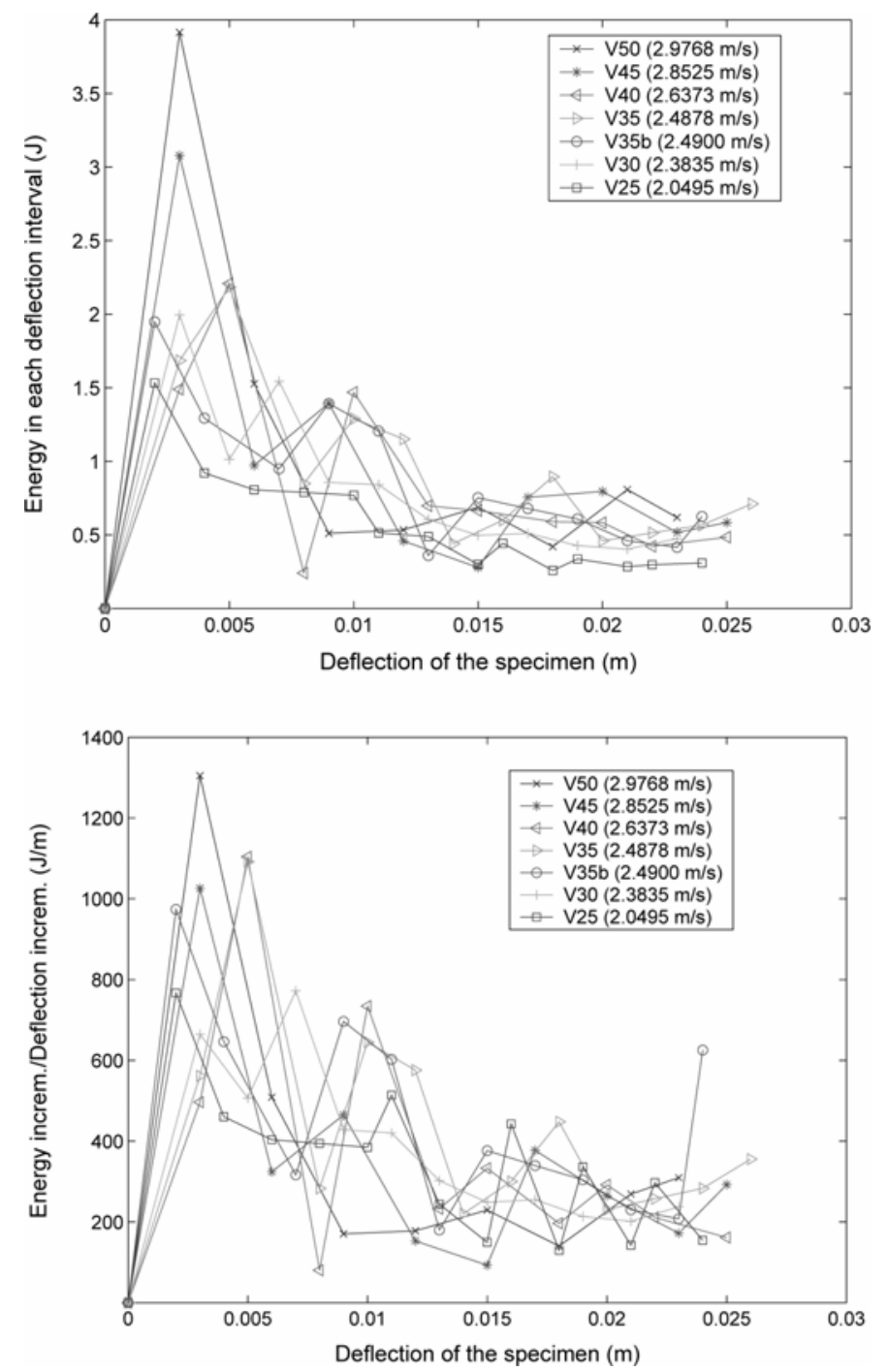

Since the second of these plots represents the incremental derivative of the evolution of the absorbed energy with respect to the deflection of the specimen, it gives us the behaviour of the slope of the curve of absorbed energy versus deflection. 
In the first of the plots of Figure 8, one observes that, following the initial peak, the curves overlap and approach a practically horizontal line.

Similar behaviour is observed in the second plot of Figure 8. After the initial peak, the curves tend to become practically horizontal when the plot is viewed at an appropriate scale, oscillating within the range 200 to $400 \mathrm{~J} / \mathrm{m}$.

These two observations merely confirm the linearity of the relationship between the accumulated energy absorbed by the specimen during the process of impact and the deflection of the specimen in that process. Indeed, this is the most important observation of the present study.

\section{Critical discussion and conclusion}

The present study corresponds to a preliminary investigation of the response of the SMC materials used in the car industry to low-energy impacts. The work has confirmed a finding which, although approximate in character, was repeated in the entire set of trials, as well as in other trials on specimens of the same material that have been carried out in the context of this same research project.

The results of the trials and their subsequent analysis can be summarised as follows:

- No clear correspondence was found between the maximum value of the force in the impactor and the height of fall used in each trial.

- The variation in the energy absorbed by the specimen was a decreasing function of the time after impact.

- The accumulated energy absorbed by the specimen during the impact process depended linearly on the deflection of the specimen in that process.

- The above finding was confirmed by the curves of the energy absorbed by the specimen in each interval of the sequence of images versus the deflection of the specimen and by the curves of the energy absorbed in each interval of the sequence divided by the incremental deflection in that interval versus the deflection of the specimen. The confirmation was shown by the two curves tending to become almost horizontal for intermediate and high deflections relative to those reached in the trials.

Crack initiation is an important mechanism, however, the focal point of this research is to define an energy level of low-speed impact due to the area of application. A SMC panel with internal damage does not pose a threat to the integrity of a truck cab for instance. What is more important for the manufacturer (and owner/user of the truck) is the visual appearance of the panels. It has been shown that the SMC with low-speed impact behaves linearly and thus is quasistatic; that is, the damage depends fundamentally on the deflection and not of the speed of the impact.

\section{Acknowledgements}

This study is part of research being carried out in collaboration with SCANIA. The authors wish to express their gratitude to Spain's Ministerio de Educación y Ciencia for partial support granted through project DPI2002-02841 of the Plan Nacional I+D+i. 


\section{References}

Abrate, S. (1994) 'Impact on laminated composites', Applied Mechanics Reviews, Vol. 47, pp.517-544.

Abrate, S. (2001) 'Modeling of impacts on composite structures', Composite Structures, Vol. 51, pp.129-138.

Aslan, Z., Karakuzu, R. and Okutan, B. (2003) 'The response of laminated composites plates under low-velocity impact loading', Composite Structures, Vol. 59, pp.119-127.

Corbett, G.G., Reid, S.R. and Johnson, W. (1996) 'Impact loading of plates and shells by free-flying projectiles: a review', International Journal of Impact Engineering, Vol. 18, No. 2, pp.141-230.

Dear, P.D. and Brown, S.A. (2003) 'Impact damage processes in reinforced polymeric materials', Composite Structures, Vol. 34, pp.411-420.

Jiang, D. and Shu, D. (2004) 'Stress distribution in damaged composite laminates under transverse impact', Composite Structures, Vol. 63, pp.407-415.

Lee, S.M., Cheon, J.S. and Im, Y.T. (1999) 'Experimental and numerical study of the impact behavior of SMC plates', Composite Structures, Vol. 47, pp.551-561.

Marsh, G. (2003) 'Composites on the road to the big time?' Reinforced Plastics, pp.33-47.

Morozov, E.V., Morozov, K.E. and Selvarajalu, V. (2003) 'Progressive damage modelling of SMC composite materials', Composite Structures, Vol. 62, pp.361-366.

Oldenbo, M., Fernberg, S.P. and Berglund, L.A. (2003) 'Mechanical behaviour of SMC composites with toughening and low density additives', Composites: Part A, Vol. 34, pp.875-885.

Richardson, M.O.W. and Wisheart, M.J. (1996) 'Review of low-velocity impact properties of composite materials', Composites Part A, Vol. 27, pp.1123-1131.

\section{Nomenclature}

$M \quad$ Mass of impactor $[\mathrm{kg}]$

$F(t) \quad$ Vertical force transmitted to the specimen in function of time [N]

$y(t) \quad$ Vertical distance in function of time $[\mathrm{m}]$

$g \quad$ Coefficient of gravity $\left(9.81 \mathrm{~m} / \mathrm{s}^{2}\right)$

$E_{c}(t) \quad$ Kinetic energy in function of time[J]

$E_{p}(t) \quad$ Potential energy in function of time[J]

$f \quad$ deflection $[\mathrm{m}]$

$E_{\text {abs }} \quad$ Absorbed energy in Joules [J] 